\title{
Hierarchical Coordination of a Hybrid AC/DC SmartGrid with Central/Distributed Energy Storage
}

\author{
Pablo Arboleya, Cristina Gonzalez-Moran \\ Pablo Garcia, Jorge Garcia and Bassam Mohamed, \\ Department of Electrical Engineering \\ University of Oviedo \\ Gijon, Spain \\ arboleyapablo@uniovi.es, gonzalezmorcristina@uniovi.es \\ garciafpablo@uniovi.es, garciajorge@uniovi.es, mohamedbassam@uniovi.es
}

\begin{abstract}
This work describes a hybrid AC/DC Smart Grid distribution scheme installed at LEMUR microgrid laboratory. The control of the microgrid is carried out according to a hierarchical coordination considering the high level control. The configuration includes a microgrid $(\mu G)$ connected to the main utility grid (MUG) by means of a solid state transformer (STT). The $\mu \mathbf{G}$ is formed by several nanogrids ( $n$ Gs). All ( $n$ Gs) are based on four wire configurations, as they are usually employed in AC distribution systems. However, the scheme is considered as a hybrid Smart Grid because the connection among different nanogrids and with the SST are DC connections. The SST is also equipped with a third port connected to a central energy storage system (CESS). The coordination between the different involved in the systems: the installed dispersed generators at nanogrid level, the nanogrids, the SST and the CESS has been implemented using a bottom-up hierarchical approach. Several configurations at nanogrid and microgrid levels are shown and analyzed. For making the coordination of the different elements of the microgrid, a fast power flow alorithm for estimating the state of the microgrid in real time was developed. In this paper the proposed structure is described paying special attention to the power flow algorithm. The results obtained with the power flow algorithm in simulations were validated at laboratory level.
\end{abstract}

\section{INTRODUCTION AND MOTIVATION}

Many power systems have beed traditionally fed by DC sources. Among them, we can find spacecrafts [1], telecommunications and data centres [2], traction systems [3] or shipboard power systems [4]. Recently, many authors have proposed different solutions for moving towards DC distribution systems [5], [6] while others proposed hybrid AC/DC systems. For instance, the topology of [7] is connected to the main utility grid through a power converter and it has two levels; the first one is an AC level, where only some distributed generators are connected and there is no load. The second one is a DC level and it is connected to the first one through an AC/DC converter. In [8] a hybrid scheme is implemented. In this particular case the conventional AC system inside a building coexists with a DC bus where the

This work was partially supported by the Spanish Ministry of Science and Innovation under Grant ENE2013-44245-R (MICROHOLO Development of a Holistic and Systematical Approach to AC Microgrids Design and Management) distributed generators and the storage systems are connected. In the present work, a hybrid distribution scheme is proposed. The authors did not use conventional power transformers. Instead, the microgrid $(\mu \mathrm{G})$ is connected to the main utility grid (MUG) by means of a three-port solid state transformer (SST) [9], [10]. With this solution, the MUG, the $\mu \mathrm{G}$, the central energy storage system (CESS) and the DC distribution system (DCDS) are simultaneously interconnected. The great advantage of SSTs is that they are much lighter and compact than conventional power transformers, so they are very suitable for a spread use in distribution systems. Multiple buildings are grouped forming different $\mathrm{AC}$ nanogrids (nGs) connected to the DCDS through DC/AC converters. Each building can be also equipped with its own energy storage system, renewable generation (the authors consider renewal generators as non dispatchable) and other dispatchable generators. The proposed scheme is depicted in Fig. 1. In next section the hybrid scheme and the bottom-up hierarchical control employed for coordinate all the resources in the microgrid is explained. Then, the paper is focused in the power flow algorithm developed by the authors to be running in real time in the microgrid control. To investigate the scale effect, the scheme will be implemented and solved in a microgrid scheme with six different nanogrids. The power flow solver was validated in the LEMUR Lab microgrid containing two nanogrids [11]. In Fig. 2, the SCADA system developed for the laboratory microgrid is shown.

\section{DISTRIBUTION SCHEME AND PROPOSED CONTROL}

The general scheme for the proposed topology in Fig. 1 can be described as follows: the whole $\mu \mathrm{G}$ is formed by a number $n$ of nGs. Each $\mathrm{nG}$ consists on a radial four-wire AC distribution feeder to which several buildings are connected. Each node represents a building labeled as $N_{i}^{j}$ where the super index $j$ is the node number inside the $\mathrm{nG}$ labeled as $i$. In general, the subindex expresses the $\mathrm{nG}$ code, and the superindex expresses the device code inside that specific $\mathrm{nG}$. Each building includes a renewable (non dispatchable) generator $(\mathrm{RG})$, a dispatchable generator (DG) and an energy storage system (ES). The character "C" after an acronym means 


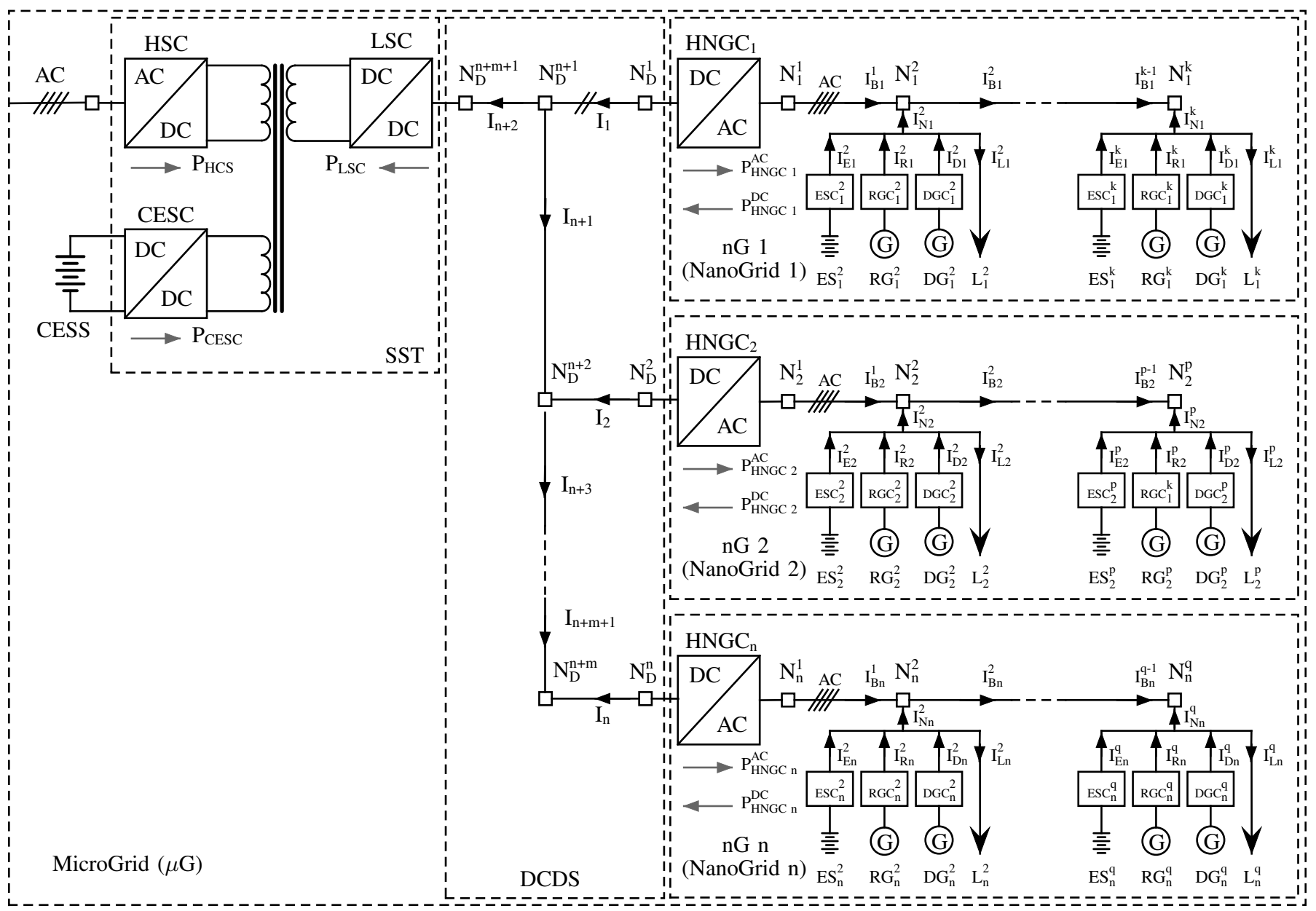

Fig. 1: General scheme of the Hybrid AC/DC Microgrid.

"Converter". For example, $\mathrm{RGC}_{1}^{2}$ is the converter interface of the renewable generator installed at building 2 of $n G 1$. The loads inside a building (L) are emulated with programable loads [12]. For programming the loads, the authors employed two kind of data: on the one hand real data provided by an electrical company and on the other hand simulated data obtained through the GENMIX software package. [13]. For describing the different element controls a bottom-up approach will be followed, starting at $\mathrm{nG}$ level. Because of they are non despatchable, the RGCs will inject the maximum available power with a unity power factor. In the case of ESCs and the DGCs, three inverse P-V droops will be implemented for each of them. The phase voltages of the three phases are read and three active power references are generated. All the droops have a dead band around the rated voltage. For instance, for a $\mathrm{ES}$, if the voltage rises or drops beyond the dead band the ESC will absorb or inject active power in the network according to its droop characteristic (see Fig. 1a). The DG injected power cannot vary uniformly from zero to its maximum (see Fig.1b). If a DGC is connected, it will develop at least its minimum power, and once it is connected, it will injected that power at least for an specific period.
The ESCs and the DGCs can independently control the active power injected to each phase, and both of them use a unity power factor. The conventional P-f droop characteristic [14] has been replaced by the characteristic P-V [15]. At typical distribution voltage levels ( $400 \mathrm{~V}$ phase to phase), the lines are nearly purely resistive, and the voltage drop is mostly derived from line resistances. The P-Vs characteristics allows the voltage regulation to be carried out in all phases independently (sort of collaborative way) by all ESCs and DGCs. Each $\mathrm{nG}$ is fed by an AC/DC converter labeled as head nanogrid converter (HNGC). This converter regulates the voltage and the frequency acting as a kind of slack node for each nG. It is a bidirectional converter, so it can inject the $\mathrm{nG}$ active power surpluses into the DCDS or extract power from the DCDS when the $\mathrm{nG}$ demands it. All the required reactive power inside the $\mathrm{nG}$ will be provided by its HNGC. In the laboratory microgrid, two 4-wires 50kVA HGNC have been installed switching at $10 \mathrm{kHz}$.

The DCDS is fed by the SST low side converter (LSC) that fixes the DCDS voltage. The DCDS rated voltage is $750 \mathrm{~V}$. The DC distribution system allows the energy exchange between nGs, the CESS and the MUG. In the SST management system, two working modes have been implemented. The first one is 


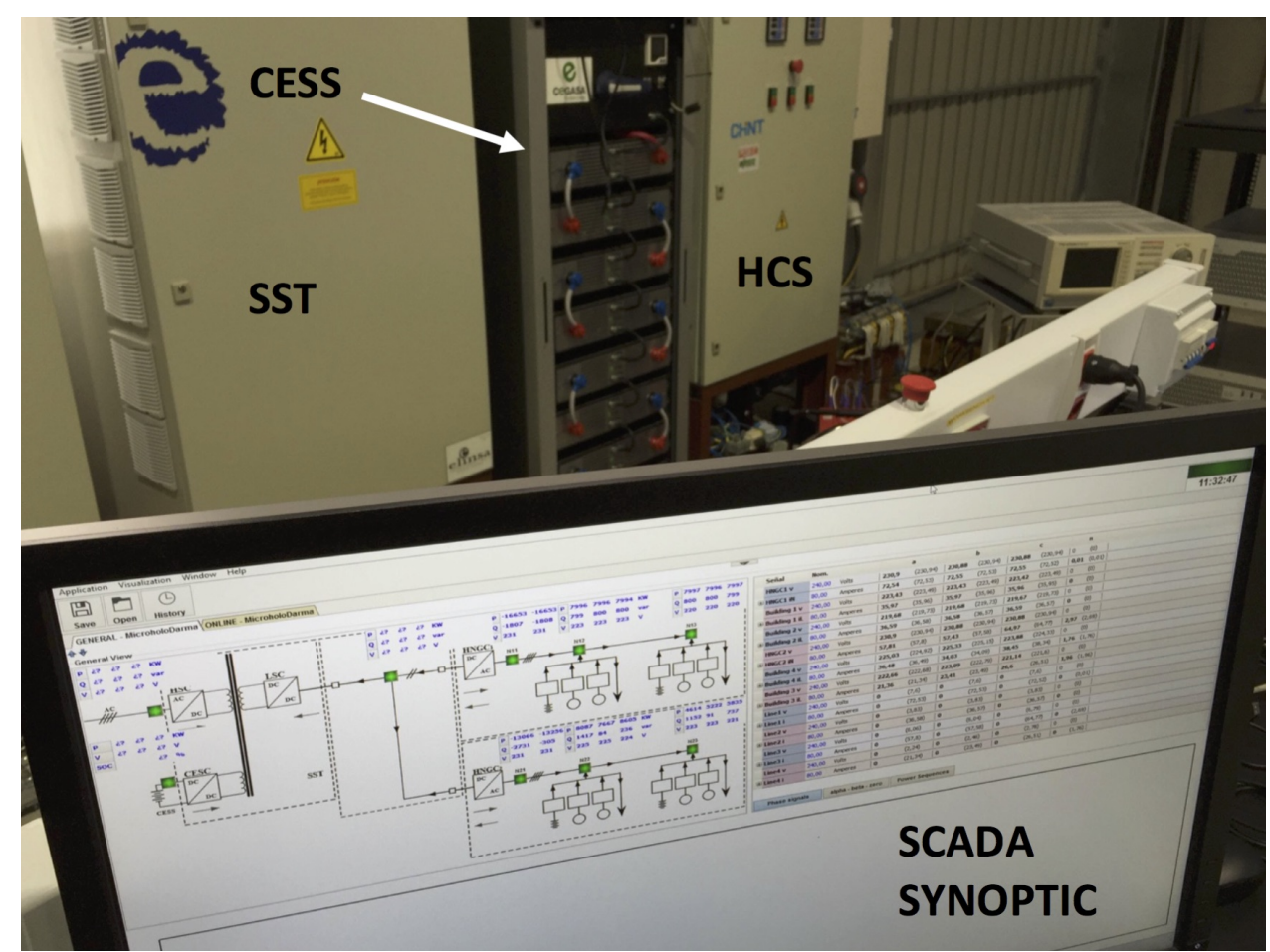

Fig. 2: SCADA system with the synoptic of the laboratory microgrid. At the background, the Central Energy Storage System (CESS) as well as the Solid State Transformer (SST) can be observed.

called Dispatchable mode. The SST high side converter (HSC) fixes the power injected or demanded from the MUG, and the power at the LSC depends on the power balance between the nGs. The CESS will compensate the power excess or shortfall. The HCS is a 150kVA converter with 6 legs and 3 wires switching at $10 \mathrm{kHz}$ with DC voltage of $900 \mathrm{~V}$. The 150kVA SST has switching frequency of $20 \mathrm{kHz}$, the Li-Ion CESS connected at the third port of the SST has a rated power of $48 \mathrm{~kW}$ and a capacity of $16 \mathrm{kWh}$ with a DC voltage of $375 \mathrm{~V}$. The second mode is called Back-up. Again the power at the LSC converter is determined by the nGs behaviour. The CESC calculates its power reference in order to keep the level of charge of the CESS among the specified limits. In this case the power unbalance is covered by the MUG through the HSC. The details about the communications among the different agents of the microgrid as well as the data processing and storage can be found in [11]. In the present work, the authors assumed 6 different nanogrids connected to the DCDS. The voltage and the current will be measured at each node of the microgrid and a distributed real time signal processing will be carried out in the frequency domain in order to obtain the sequence components (positive/negative/zero). The distributed signal processors will have a communication link with the central server at a sampling rate of $200 \mathrm{~ms}$ allowing a bandwidth of $60 \mathrm{~kb} / \mathrm{s}$. The power flow algorithms used in this abstract is adapted to run a state estimator at the central server level in which the control of the whole microgrid will be implemented.

\section{PF SOLVER}

The PF algorithm is based in Backward/Forward Sweep method. The formulation uses the conventional $a b c$ reference frame and is based on the node incidence matrix $\Gamma$. In this matrix, each row represent a line and each column a node. The element $\Gamma_{i j}$ is 1 when the tail of the branch $\mathrm{i}$ is node $\mathrm{j}$, and the element $\Gamma_{i j}$ is -1 when the head of the branch $i$ is node j. $\Gamma_{i j}$ will be 0 otherwise. Using this matrix formulation, all Kirchhoff voltage and current laws in the $\mathrm{nG}$ can be expressed

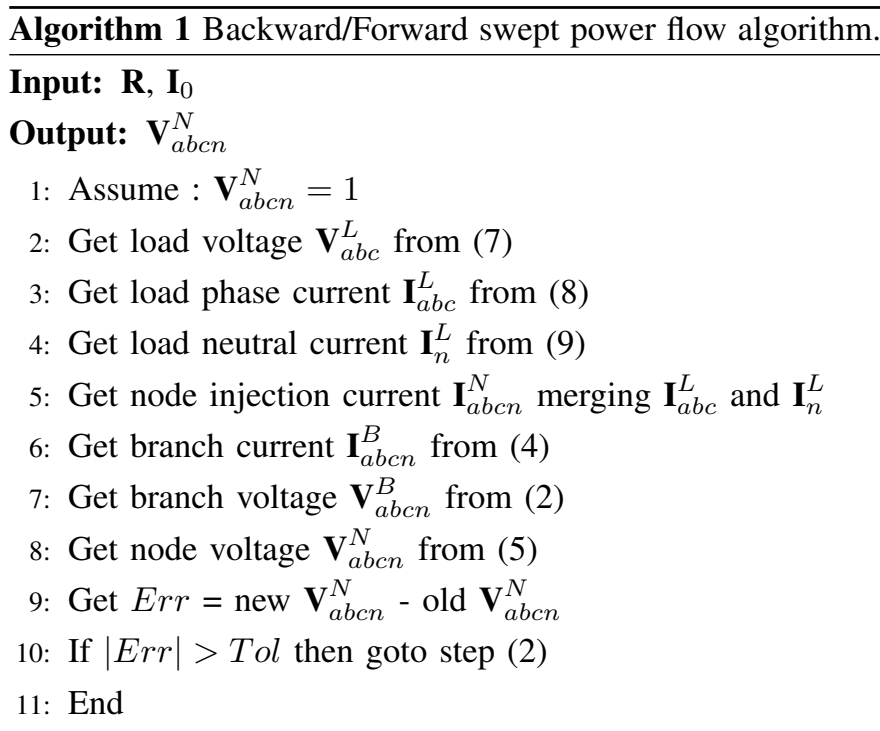




\begin{tabular}{|c|c|c|c|c|c|c|c|c|c|c|c|c|c|c|c|}
\hline \multicolumn{8}{|c|}{ nG1 } & \multicolumn{8}{|c|}{ nG4 } \\
\hline \multicolumn{2}{|c|}{$V_{a}\left(p . u .,^{\circ}\right)$} & \multicolumn{2}{|c|}{$V_{b}\left(p . u .,^{\circ}\right)$} & \multicolumn{2}{|c|}{$V_{c}\left(p . u .,^{\circ}\right)$} & \multicolumn{2}{|c|}{$V_{n}\left(p . u .,^{\circ}\right)$} & \multicolumn{2}{|c|}{$V_{a}\left(p . u .{ }^{\circ}\right)$} & \multicolumn{2}{|c|}{$V_{b}\left(p . u .,^{\circ}\right)$} & \multicolumn{2}{|c|}{$V_{c}\left(p . u .,^{\circ}\right)$} & \multicolumn{2}{|c|}{$V_{n}\left(p . u .,^{\circ}\right)$} \\
\hline mag. & ang. & mag. & ang. & mag. & ang. & mag. & ang. & mag. & ang. & mag. & ang. & mag. & ang. & mag. & ang. \\
\hline 0.93 & 0.4 & 0.95 & -120.1 & 0.88 & 105.6 & 0.05 & 105.6 & 0.92 & 0.2 & 0.95 & -119.8 & 0.94 & 120.5 & 0.03 & 15.3 \\
\hline 0.88 & 0.8 & 0.92 & -120.2 & 0.80 & 102.1 & 0.90 & 102.1 & 0.86 & 0.3 & 0.93 & -119.6 & 0.90 & 120.8 & 0.06 & 21.4 \\
\hline 0.84 & 1.2 & 0.91 & -120.3 & 0.76 & 94.8 & 0.11 & 94.8 & 0.82 & 0.6 & 0.91 & -119.5 & 0.89 & 121.1 & 0.90 & 15.1 \\
\hline \multicolumn{2}{|c|}{$I_{a}\left(A,{ }^{\circ}\right)$} & \multicolumn{2}{|c|}{$I_{b}\left(A,^{\circ}\right)$} & \multicolumn{2}{|c|}{$I_{c}\left(A,{ }^{\circ}\right)$} & \multicolumn{2}{|c|}{$I_{n}\left(A,{ }^{\circ}\right)$} & \multicolumn{2}{|c|}{$I_{a}\left(A,{ }^{\circ}\right)$} & \multicolumn{2}{|c|}{$I_{b}\left(A,{ }^{\circ}\right)$} & \multicolumn{2}{|c|}{$I_{c}\left(A,{ }^{\circ}\right)$} & \multicolumn{2}{|c|}{$I_{n}\left(A,{ }^{\circ}\right)$} \\
\hline mag. & ang. & mag. & ang. & mag. & ang. & mag. & ang. & mag. & ang. & mag. & ang. & mag. & ang. & mag. & ang. \\
\hline 75.46 & -20.5 & 85.43 & -136.0 & 115.63 & 102.5 & 314.40 & -59.6 & 79.96 & -18.5 & 98.39 & -136.8 & 71.87 & 96.2 & 21.57 & 80.3 \\
\hline 35.94 & -22.8 & 45.84 & -135.0 & 116.47 & 103.8 & 72.00 & -68.0 & 62.55 & -12.9 & 34.52 & -146.7 & 78.74 & 103.9 & 45.47 & -106.9 \\
\hline 121.26 & -24.2 & 38.86 & -134.9 & 136.40 & 105.1 & 72.37 & -131.2 & 117.20 & -23.2 & 33.51 & -144.9 & 40.51 & 77.7 & 92.63 & 163.8 \\
\hline $\begin{array}{c}P_{a} \\
(\mathrm{~kW})\end{array}$ & $\begin{array}{c}Q_{a} \\
\text { (kVAr) }\end{array}$ & $\begin{array}{c}P_{b} \\
(\mathrm{~kW})\end{array}$ & $\begin{array}{c}Q_{b} \\
\text { (kVAr) }\end{array}$ & $\begin{array}{c}P_{c} \\
(\mathrm{~kW})\end{array}$ & $\begin{array}{c}Q_{c} \\
(\mathrm{kVAr})\end{array}$ & $\begin{array}{c}P_{T} \\
(\mathrm{~kW})\end{array}$ & $\begin{array}{c}Q_{T} \\
(\mathrm{kVAr})\end{array}$ & $\begin{array}{c}P_{a} \\
(\mathrm{~kW})\end{array}$ & $\begin{array}{c}Q_{a} \\
\text { (kVAr) }\end{array}$ & $\begin{array}{c}P_{b} \\
(\mathrm{~kW})\end{array}$ & $\begin{array}{c}Q_{b} \\
\text { (kVAr) }\end{array}$ & $\begin{array}{c}P_{c} \\
(\mathrm{~kW})\end{array}$ & $\begin{array}{c}Q_{c} \\
\text { (kVAr) }\end{array}$ & $\begin{array}{c}P_{T} \\
(\mathrm{~kW})\end{array}$ & $\begin{array}{c}Q_{T} \\
(\mathrm{kVAr})\end{array}$ \\
\hline 11.00 & 3.60 & 13.00 & 4.20 & 15.00 & 4.90 & 39.00 & 12.70 & 11.00 & 3.60 & 15.00 & 4.20 & 10.00 & 4.90 & 36.00 & 12.70 \\
\hline 5.00 & 1.64 & 7.00 & 2.30 & 13.00 & 4.20 & 25.00 & 8.14 & 8.00 & 1.64 & 5.00 & 2.30 & 11.00 & 4.20 & 24.00 & 8.14 \\
\hline 16.00 & 5.25 & 6.00 & 1.97 & 14.00 & 4.60 & 36.00 & 11.82 & 13.00 & 5.25 & 5.00 & 1.97 & 4.00 & 4.60 & 22.00 & 11.82 \\
\hline \multicolumn{8}{|c|}{ nG2 } & & & & $\mathbf{n}$ & & & & \\
\hline$V_{a}$ &.$\left.{ }^{\circ}\right)$ & $V_{b}($ & $\left.u .,^{\circ}\right)$ & $V_{c}$ &.$\left.{ }^{\circ}\right)$ & $V_{n}$ & $\left.u \cdot .^{\circ}\right)$ & $V_{a}$ & $\left.u .{ }^{\circ}\right)$ & $V_{b}$ & $\left.u .{ }^{\circ}\right)$ & $V_{c}$ & $\left.u .,^{\circ}\right)$ & $V_{n}$ & $\left.u .,^{\circ}\right)$ \\
\hline mag. & ang. & mag. & ang. & mag. & ang. & mag. & ang. & mag. & ang. & mag. & ang. & mag. & ang. & mag. & ang. \\
\hline 1.04 & 2.0 & 0.94 & -120.2 & 0.94 & 121.1 & 0.09 & -160.2 & 1.04 & 1.9 & 1.02 & -118.6 & 1.01 & 121.9 & 0.01 & 164.9 \\
\hline 1.07 & 3.2 & 0.94 & -120.3 & 0.90 & 122.0 & 0.14 & -163.6 & 1.06 & 3.0 & 1.04 & -117.8 & 1.02 & 123.0 & 0.02 & 157.2 \\
\hline 1.09 & 3.9 & 0.90 & -120.3 & 0.89 & 122.6 & 0.16 & -160.6 & 1.07 & 3.7 & 1.04 & -117.4 & 1.02 & 123.5 & 0.03 & 162.6 \\
\hline$I_{a}$ & & $I_{b}$ & $\left.1,{ }^{\circ}\right)$ & $I_{c}$ & & $I_{n}$ & $\left.4,{ }^{\circ}\right)$ & $I_{a}$ & & $I_{b}$ & $\left.1,{ }^{\circ}\right)$ & $I_{c}$ & $\left.1,,^{\circ}\right)$ & $I_{n}$ & $\left.4,,^{\circ}\right)$ \\
\hline mag. & ang. & mag. & ang. & mag. & ang. & mag. & ang. & mag. & ang. & mag. & ang. & mag. & ang. & mag. & ang. \\
\hline 62.83 & -158.5 & 109.39 & -132.1 & 73.55 & 89.6 & 134.86 & 13.1 & 61.95 & 168.0 & 39.27 & 102.2 & 42.79 & -14.3 & 25.06 & -12.0 \\
\hline 41.56 & -163.8 & 41.19 & -138.4 & 82.16 & 92.6 & 85.99 & -30.1 & 49.49 & 39.2 & 32.56 & 88.1 & 40.42 & -18.2 & 14.85 & -140.8 \\
\hline 69.19 & -152.1 & 41.97 & -134.3 & 42.83 & 63.4 & 75.25 & 18.7 & 86.93 & 159.1 & 31.66 & 85.6 & 28.97 & 20.2 & 26.08 & -20.9 \\
\hline $\begin{array}{c}P_{a} \\
\mathrm{~kW}\end{array}$ & $\begin{array}{c}Q_{a} \\
\text { kVAr }\end{array}$ & $\begin{array}{c}P_{b} \\
\mathrm{~kW}\end{array}$ & $\begin{array}{c}Q_{b} \\
\text { kVAr }\end{array}$ & $\begin{array}{c}P_{c} \\
\mathrm{~kW}\end{array}$ & $\begin{array}{c}Q_{c} \\
\mathrm{kVAr}\end{array}$ & $\begin{array}{l}P_{T} \\
\mathrm{~kW}\end{array}$ & $\begin{array}{c}Q_{T} \\
\text { kVAr }\end{array}$ & $\begin{array}{c}P_{a} \\
\mathrm{~kW}\end{array}$ & $\begin{array}{c}Q_{a} \\
\mathrm{kVAr}\end{array}$ & $\begin{array}{c}P_{b} \\
\mathrm{~kW}\end{array}$ & $\begin{array}{c}Q_{b} \\
\text { kVAr }\end{array}$ & $\begin{array}{c}P_{c} \\
\mathrm{~kW}\end{array}$ & $\begin{array}{c}Q_{c} \\
\text { kVAr }\end{array}$ & $\begin{array}{l}P_{T} \\
\mathrm{~kW}\end{array}$ & $\begin{array}{c}Q_{T} \\
\text { kVAr }\end{array}$ \\
\hline-11.00 & 3.60 & 15.00 & 4.20 & 10.00 & 4.90 & 14.00 & 12.27 & -10.00 & 3.60 & -5.00 & 4.20 & -5.00 & 4.90 & -20.00 & 12.70 \\
\hline-8.00 & 1.64 & 5.00 & 2.30 & 11.00 & 4.20 & 8.00 & 8.14 & -5.00 & 1.64 & -5.00 & 2.30 & -5.10 & 4.20 & -1.51 & 8.14 \\
\hline-13.00 & 5.25 & 5.00 & 1.97 & 4.00 & 4.60 & -4.00 & 11.82 & -10.00 & 5.25 & -5.00 & 1.90 & -1.00 & 4.60 & -16.00 & 11.82 \\
\hline & & & & & & & & & & & & & & & \\
\hline$V_{a}$ & $\left.u .,^{\circ}\right)$ & $V_{b}($ & $\left.u .,^{\circ}\right)$ & $V_{c}(1$ & $\left.u .,^{\circ}\right)$ & $V_{n}$ & $\left.u .,^{\circ}\right)$ & $V_{a}$ & $\left.u .{ }^{\circ}\right)$ & $V_{b}$ & $\left.u .,^{\circ}\right)$ & $V_{c}$ & $\left.u .,^{\circ}\right)$ & $V_{n}$ & $\left.u .,^{\circ}\right)$ \\
\hline mag. & ang. & mag. & ang. & mag. & ang. & mag. & ang. & mag. & ang. & mag. & ang. & mag. & ang. & mag. & ang. \\
\hline 1.05 & 2.0 & 1.02 & -118.5 & 0.96 & 121.5 & 0.05 & 155.7 & 0.94 & -1.3 & 0.88 & -121.8 & 0.94 & 119.2 & 0.06 & -110.6 \\
\hline 1.08 & 3.2 & 1.02 & -118.1 & 1.00 & 122.7 & 0.06 & 179.8 & 0.90 & -2.4 & 0.79 & -123.3 & 0.90 & 118.7 & 0.10 & -111.2 \\
\hline 1.09 & 4.0 & 1.00 & -118.1 & 0.98 & 123.1 & 0.08 & -170.5 & 0.88 & -2.9 & 0.75 & -124.1 & 0.88 & 118.4 & 0.12 & -111.5 \\
\hline$I_{a}$ & $\left(,{ }^{\circ}\right)$ & $I_{b}$ & $\left.1,{ }^{\circ}\right)$ & $I_{c}$ & $\left(,{ }^{\circ}\right)$ & $I_{n}$ & $\left.4,{ }^{\circ}\right)$ & $I_{a}$ &,$\left.^{\circ}\right)$ & $I_{b}$ & $\left.1,{ }^{\circ}\right)$ & $I_{c}$ & $\left.4,{ }^{\circ}\right)$ & $I_{n}$ & $\left.4,{ }^{\circ}\right)$ \\
\hline mag. & ang. & mag. & ang. & mag. & ang. & mag. & ang. & mag. & ang. & mag. & ang. & mag. & ang. & mag. & ang. \\
\hline 65.10 & -161.0 & 93.68 & 79.8 & 72.02 & 93.7 & 151.20 & -70.9 & 63.70 & 1.8 & 111.40 & -122.5 & 62.66 & 116.7 & 43.49 & 55.8 \\
\hline 44.10 & -165.4 & 33.99 & 89.5 & 74.74 & -39.3 & 289.30 & 122.3 & 65.42 & 3.3 & 131.60 & -124.9 & 63.39 & 114.3 & 58.88 & 52.0 \\
\hline 73.10 & -153.6 & 34.30 & -135.8 & 39.10 & 69.7 & 79.00 & 79.0 & 65.95 & 4.1 & 145.50 & -126.6 & 63.66 & 113.0 & 70.38 & 49.6 \\
\hline $\begin{array}{c}P_{a} \\
\mathrm{~kW}\end{array}$ & $\begin{array}{c}Q_{a} \\
\text { kVAr }\end{array}$ & $\begin{array}{c}P_{b} \\
\mathrm{~kW}\end{array}$ & $\begin{array}{c}Q_{b} \\
\text { kVAr }\end{array}$ & $\begin{array}{c}P_{c} \\
\mathrm{~kW}\end{array}$ & $\begin{array}{c}Q_{c} \\
\text { kVAr }\end{array}$ & $\begin{array}{l}P_{T} \\
\mathrm{~kW}\end{array}$ & $\begin{array}{c}Q_{T} \\
\text { kVAr }\end{array}$ & $\begin{array}{c}P_{a} \\
\mathrm{~kW}\end{array}$ & $\begin{array}{c}Q_{a} \\
\text { kVAr }\end{array}$ & $\begin{array}{c}P_{b} \\
\mathrm{~kW}\end{array}$ & $\begin{array}{c}Q_{b} \\
\text { kVAr }\end{array}$ & $\begin{array}{c}P_{c} \\
\mathrm{~kW}\end{array}$ & $\begin{array}{c}Q_{c} \\
\text { kVAr }\end{array}$ & $\begin{array}{l}P_{T} \\
\mathrm{~kW}\end{array}$ & $\begin{array}{c}Q_{T} \\
\text { kVAr }\end{array}$ \\
\hline-11.00 & 3.60 & -15.00 & 4.20 & 10.00 & 4.90 & -16.00 & 12.70 & 10.00 & 0.00 & 15.00 & 0.00 & 10.00 & 0.00 & 35.00 & 0.00 \\
\hline-8.00 & 1.64 & -5.00 & 2.30 & -11.00 & 4.20 & -24.00 & 8.14 & 10.00 & 0.00 & 15.00 & 0.00 & 10.00 & 0.00 & 35.00 & 0.00 \\
\hline-13.00 & 5.25 & 5.00 & 1.97 & 4.00 & 4.60 & -4.00 & 11.82 & 10.00 & 0.00 & 15.00 & 0.00 & 10.00 & 0.00 & 35.00 & 0.00 \\
\hline
\end{tabular}

TABLE I: Test nanogrid configurations.

in a extremely simple way:

$$
\begin{array}{ll}
V_{a b c n}^{B} & =\Gamma \times V_{a b c n}^{N} \\
V_{a b c n}^{B} & =\quad Z \times I_{a b c n}^{B} \\
I_{a b c n}^{N} & =-\Gamma^{T} \times I_{a b c n}^{B} \\
I_{a b c n}^{B} & =-\left(\Gamma^{T}\right)^{-1} \times I_{a b c n}^{N} \\
V_{a b c n}^{N} & =\Gamma^{-1} \times V_{a b c n}^{B}
\end{array}
$$

Where:

- $V_{a b c n}^{B}$ is four column vector containing the voltage drop in the three phases and the neutral in all lines of the microgrid.

- $V_{a b c n}^{N}$ is a four column vector containing the voltages respect to the ground of the three phases and the neutral at all nodes of the $n G^{\prime} s$.
- $I_{a b c n}^{B}$ is a four column vector containing the currents in the three phases and the neutral in all the lines of the $n G^{\prime} s$.

- $\boldsymbol{I}_{\boldsymbol{a} \boldsymbol{a b c n}}^{\boldsymbol{N}}$ is a four column vector containing the nodal currents injected by the three phases and the neutral in all nodes of the $n G^{\prime} s$.

- $Z$ Is the impedance matrix containing the impedances of all the lines of the $n G^{\prime} s$.

- $\Gamma$ Is the above described node incidence matrix.

With the expresion (1), the voltage drops in all branches can be obtained starting from all node voltages in the whole $n G^{\prime} s$. Using the impedances of the branches and the branches voltages, the current through them can be obtained using expression (2), that represents the Kirchhoff voltage law in all branches of the $n G^{\prime} s$. The equation (3) represents the 
Kirchhoff Current law in all nodes of the $n G^{\prime} s$. The operator $\left({ }^{T}\right)$ stands for the transpose operator. It is important to remark that in a general network, the node incidence matrix is not always square, because it contains the same number of rows as lines and the same number of columns as nodes. However in our case all $n G^{\prime} s$ are radial, so we take advantage of this feature. When we work with radial networks of $N$ nodes, the number of lines is $N-1$. We remove the node representing the head nanogrid converters from each nanogrid node incidence matrix because it acts as a slack node of the $n G$. Under this circumstance, the node incidence matrix is square and regular, so from the equation (3) we can obtain directly the equation (4). And also from the eq. (1) we can obtain directly the equation (5). Each building of the nanogrid is represented as an aggregated load model characterised by the next equations.

$\begin{array}{lll}S_{a b c}^{L} & = & P_{a b c}^{L}+j \cdot Q_{a b c}^{L} \\ V_{a b c}^{L} & = & V_{a b c}^{N}-V_{n}^{N} \\ I_{a b c}^{L} & = & C o n j\left(S_{a b c}^{L} / V_{a b c}^{L}\right) \\ I_{n}^{L} & = & -\sum I_{a b c}^{L}\end{array}$

Where:

- $S_{a b c}^{L}$ is the apparent power injected or extracted from the grid in a node (each node represents a building).

- $\boldsymbol{P}_{\boldsymbol{a} b \boldsymbol{c}}^{\boldsymbol{L}}$ is the active power injected of extracted from the grid in a node.

- $\boldsymbol{Q}_{a b c}^{L}$ is the reactive power injected or extracted from the grid in a node.

- $\boldsymbol{V}_{\boldsymbol{a} b \boldsymbol{c}}^{\boldsymbol{L}}$ represents the voltage in the different phases of the building, phase to neutral voltages without considering the neutral.

- $\boldsymbol{V}_{\boldsymbol{a b c}}^{\boldsymbol{N}}$ is a vector with three positions, one per phase without considering the neutral, containing the phase to ground voltages of the nodes.

- $\boldsymbol{V}_{n}^{N}$ is vector containing the neutral to ground voltage.

- $\boldsymbol{I}_{\boldsymbol{a} \boldsymbol{b} \boldsymbol{c}}^{\boldsymbol{L}}$ is a vector with three positions containing the aggregated current through each of the phases of the building, it does not contain the neutral current.

- $\boldsymbol{I}_{\boldsymbol{n}}^{\boldsymbol{L}}$ is a vector containing the aggregated neutral current of the building.

It must be remarked that all the loads and appliances of the building work connected between the phase and the neutral. In this case we considered a Wye connection equivalent for the aggregated building for the sake of simplicity but without loosing any generality. All the voltages were defined previously between the phases and the ground, so before making the power calculations they must be converted to phase to neutral voltages. Another important thing that must be considered is that in this particular case of study, the lengths of the lines are quite short so the neutral is just grounded at head nanogrid converter (HNGC) level. The neutrals are not grounded at building level, so in this case the Kron reduction can not be applied to the Carson's equations and the neutral voltage con not be assumed as zero in the nodes of the nanogrids [16]. This feature of the problem can create multiple convergence problems in power flow algorithms when the unbalance of the load produce non-neglectable neutral voltage values. The above presented equations are the ones

\begin{tabular}{l|cccccc}
$\mathbf{n G}$ & $\mathbf{1}$ & $\mathbf{2}$ & $\mathbf{3}$ & $\mathbf{4}$ & $\mathbf{5}$ & $\mathbf{6}$ \\
\hline \hline Pa (kW) & 35.01 & -26.05 & -27.71 & 39.93 & -16.89 & 31.80 \\
$\mathbf{P b}(\mathbf{k W})$ & 26.77 & 30.49 & -13.83 & 25.38 & -14.10 & 63.19 \\
$\mathbf{P c}(\mathbf{k W})$ & 57.80 & 26.12 & 3.20 & 27.99 & -10.61 & 30.83 \\
\hline $\mathbf{Q a}(\mathbf{k W})$ & 14.71 & 10.94 & 10.58 & 13.97 & -12.28 & -1.71 \\
$\mathbf{Q b}(\mathbf{k W})$ & 7.41 & 7.56 & 9.44 & 9.48 & 9.05 & 5.32 \\
$\mathbf{Q c}(\mathbf{k W})$ & 16.71 & 18.09 & 14.44 & 12.75 & 14.06 & 2.88 \\
\hline \hline
\end{tabular}

TABLE II: Nanogrids active and reactive power from the $\operatorname{slack}(\mathrm{HNGC})$.

used by the developed power flow solver. The pseudocode of the solver can be observed in Alg. 1. As it can be observed is a Backward/Foward swept based algorithm. It starts assuming a plain voltage profile of 1 p.u. in all nodes (rated voltages and angles in all phases and zero voltage in all neutral points). Then using the expression (7) it obtains the phase to neutral voltages in all buildings. With these voltages and assuming as known variable the apparent power consumed by all buildings it calculates the phase current in all buildings using the expression (8). With the currents consumed by all buildings in all phases the neutral currents of the whole grid can be calculated using eq. (9). Merging the vectors with the phase currents and the neutral currents in all buildings we get the nodal current vector. With the nodal currents in all nodes, the currents through all lines can be easily obtained using eq. (4), and with the line currents we obtain the line voltage drops by means of the Kirchhoff voltage laws (eq. (2). With the voltage drops in all lines, the nodal voltages are obtained using the expression (5), and finally the obtained nodal voltages are compared with the ones of the previous iteration defining a tolerance as stop criteria.

\section{RESUlTS}

Different configurations at nanogrid and microgrid levels have been studied. For building the different studied cases, six different nanogrids were considered. Each of them was formed by three buildings. All HNGC converters act as slack nodes from the nanogrids point of view. The lines that feed the buildings are four wire ( 3 phases plus neutral) configurations. For the studied cases, the lines were modeled as typical distribution lines (short lines with $R=0.1 \Omega / \mathrm{km}$ and $L=0.1 \mathrm{H} / \mathrm{km})$. There are $500 \mathrm{~m}$ between building in a nanogrid, resulting in three lines of $500 \mathrm{~m}$ per nanogrid. As it is was depicted in Fig. 1, the buildings are connected in series. In these cases the active and reactive powers per phase and per building are known. The input powers, as well as the obtained power flow results in each nanogrid are shown in Table I. The active or reactive powers per phase and per building are obtained by adding the demanded powers as positive terms and the generated powers as negative terms, so in the table, a positive value per phase (active or reactive) indicates that there is more demand than generation in that building in that phase. A negative value means there is more generation, so the 


\begin{tabular}{c|l|l|l} 
configuration & activated nGs & Q HNGCi (kVAr) & P LSC (kW) \\
\hline \hline 1 & 146 & $38.83,36.20,06.49$ & 338.72 \\
\hline 2 & 123456 & $38.83,36.59,34.46,36.20,10.83,06.49$ & 289.33 \\
\hline 3 & 2356 & $36.59,34.46,10.83,06.49$ & 76.45 \\
\hline 4 & 246 & $36.59,36.20,06.49$ & 249.69 \\
\hline 5 & 1356 & $38.83,34.46,10.83,06.49$ & 165.47 \\
\hline 6 & 256 & $36.59,10.83,06.49$ & 114.79 \\
\hline 7 & 23456 & $36.59,34.46,36.20,10.83,06.49$ & 169.75 \\
\hline 8 & 1234 & $38.83,36.59,34.46,36.20$ & 205.1 \\
\hline 9 & 235 & $36.59,34.46,10.83$ & -49.39 \\
\hline 10 & 35 & $34.46,10.83$ & -79.95 \\
\hline \hline
\end{tabular}

TABLE III: Microgrid configurations.

surplus power is sent to the $n G$. These $P_{a}, P_{b}$ and $P_{c}$ values include the load demand, the injected or consumed ESC power, the generated power in the RG unit and the generated power in the DG unit. (constant power models in these cases) In the table the voltages at each node are also shown (per unit system in a $400 \mathrm{~V}$ phase to phase voltage base). The currents are also calculated, but in this case the values are shown in Amperes, peak values per phase. The cases were randomly selected, in order to consider close to real situations. Once the power flow was solved for all nanogrid configurations (results in Table I), the second step in the power flow algorithm consists on solving the power flow from the slack point of view. The total active and reactive powers that the slack has to fed to the nanogrids in all cases and per phase for the given configurations form 1 to 6 are shown in Table II. There are some unexpected results in that Table. For example, for nanogrid 6 there is some reactive power consumption in phases $\mathrm{b}$ and $\mathrm{c}(5.32 \mathrm{~kW}$ and $2.88 \mathrm{~kW})$, and there is also some reactive power generation at phase a $-1.71 \mathrm{kVAr}$, although there is no reactive power consumption or generation in the buildings. It is easy to understand that the reactive power is consumed in the lines, due to its inductive characteristic, but it is not very common to obtain a reactive power generation in a inductive line. The reason is the shifting of the neutral point that appears in ungrounded and unbalanced systems. This will be a typical case in nanogrids formed by buildings, because the neutral point in the load is not grounded in a building. A more detailed inspection in the Table would reveal other similar cases. Another example can be seen in nanogrid 1 , in which the total reactive power demanded in phase b (Table I: $4.2+2.3+1.97=8.47 \mathrm{kVAr}$ ) is higher than the generated power from the slack in that phase (Table II: $7.41 k V A r)$. The last step in the power flow solution is the active power exchanged between the slack (divided into different HNGCs) and the LSC connected to one of the windings of the SST. There is no reactive power exchange at this step because the reactive power demanded or provided at nanogrid level is delivered or absorbed from the HNGC of the nanogrid, and the connection between HNGCs and the LSC is a DC link. 10 different study cases were defined. Each of them is a different configuration for the microgrid in which some nanogrids are included. Table III summarizes the considered cases and the obtained results. For instance, in case 5 only nanogrids $1,3,5$ and 6 are connected to the LSC.
The active power $P_{L S C}$ is positive in this case. That means the microgrid is demanding active power from the LSC to the nanogrids. This active power can be fed from the CESS or from the AC main grid, depending on the charging level and the specifications of the LSC (this upper level is beyond the aim of this paper). There are some configurations in which the surplus powers in a nanogrid can be consumed in other nanorids in a way that the LSC is delivering a minimun amount of power (for instance, configuration 3). In cases 9 and 10 the nanogrids are delivering active power to the LSC side, so the surplus power can be used to charge the CESS, in case of need or can be injected to the AC main grid if the CESS is already full.

\section{CONCLUSions}

The hybrid AC/DC Smart Grid scheme used at LEMUR laboratory microgrid has been described. In the proposed scheme, a nanogrid has been defined as a set of buildings and a microgrid has been defined as a set of nanogrids. Each building includes loads, a dispatchable generator, a nondispatchable generator (based in renewal generators) and a energy storage system. The net active and reactive powers at building level are considered as the net powers resulting from the addition of all the included elements per phase, but at nanogrid level the active and reactive powers at the slack cannot be directly obtained by adding the powers of the buildings of the nanogrids. This is due to the shifting of the neutral point that appears in ungrounded and unbalanced systems for four wire configurations. This issue has been demonstrated with several power flow calculations at nanogrid and microgrid levels carried out with the BFS power flow solver developed for running in real time in the microgrid central control. The power flow solution at nanogrid and microgrid levels gives the inputs for the power flow approach from the viewpoint of the SST, because the active power $P_{L S C}$ is obtained. The accuracy of the proposed algorithm has been validated with real measurements in the laboratory set up.

\section{REFERENCES}

[1] J. A. Momoh, K. D'Arnaud, and W. Zheng, "Spacecraft ring-bus power system management scheme," in IEEE PES General Meeting, July 2010 , pp. 1-6. 
[2] E. Candan, P. S. Shenoy, and R. C. N. Pilawa-Podgurski, "A seriesstacked power delivery architecture with isolated differential power conversion for data centers," IEEE Transactions on Power Electronics, vol. 31, no. 5, pp. 3690-3703, May 2016

[3] P. Arboleya, B. Mohamed, C. Gonzlez-Morn, and I. El-Sayed, "Bfs algorithm for voltage-constrained meshed dc traction networks with nonsmooth voltage-dependent loads and generators," IEEE Transactions on Power Systems, vol. 31, no. 2, pp. 1526-1536, March 2016.

[4] A. Ghareeb, A. Mohamed, and O. Mohammed, "DC microgrids and distribution systems: An overview," in Power and Energy Society General Meeting (PES), 2013 IEEE, July 2013, pp. 1-5.

[5] L. Che and M. Shahidehpour, "DC microgrids: Economic operation and enhancement of resilience by hierarchical control," Smart Grid, IEEE Transactions on, vol. 5, no. 5, pp. 2517-2526, Sept 2014.

[6] D. Ricchiuto, R. Mastromauro, M. Liserre, I. Trintis, and S. MunkNielsen, "Overview of multi-DC-bus solutions for DC microgrids," in Power Electronics for Distributed Generation Systems (PEDG), 2013 4th IEEE International Symposium on, July 2013, pp. 1-8.

[7] N. Eghtedarpour and E. Farjah, "Power control and management in a hybrid AC/DC microgrid," Smart Grid, IEEE Transactions on, vol. 5, no. 3, pp. 1494-1505, May 2014.

[8] P. Arboleya, C. Gonzalez-Moran, M. Coto, M. Falvo, L. Martirano, D. Sbordone, I. Bertini, and B. Pietra, "Efficient energy management in smart micro-grids: ZERO grid impact buildings," Smart Grid, IEEE Transactions on, vol. 6, no. 2, pp. 1055-1063, March 2015.

[9] X. Yu, X. She, X. Zhou, and A. Q. Huang, "Power management for dc microgrid enabled by solid-state transformer," IEEE Transactions on Smart Grid, vol. 5, no. 2, pp. 954-965, March 2014.

[10] D. Shah and M. L. Crow, "Online volt-var control for distribution systems with solid-state transformers," IEEE Transactions on Power Delivery, vol. 31, no. 1, pp. 343-350, Feb 2016.

[11] P. Garcia, P. Arboleya, B. Mohamed, A. Vega, and M. Vega, "Implementation of a hybrid distributed/centralized real-time monitoring system for a dc/ac microgrid with energy storage capabilities," IEEE Transactions on Industrial Informatics, vol. PP, no. 99, pp. 1-1, 2016.

[12] D. Novak, M. Beraki, G. Villa, and P. Garcia, "Low-cost programmable three phase load for microgrids labs," in Environment and Electrical Engineering (EEEIC), 2015 IEEE 15th International Conference on June 2015, pp. 599-604.

[13] P. Arboleya, E. Dominguez, and F. Lorenzo, "Unified generating and storing capacity reliability evaluation in nearly-zero energy buildings," in Power and Energy Society General Meeting, July 2015. IEEE, July 2015, pp. $1-5$.

[14] P. Arboleya, D. Diaz, J. Guerrero, P. Garcia, F. Briz, C. GonzalezMoran, and J. G. Aleixandre, "An improved control scheme based in droop characteristic for microgrid converters," Electric Power Systems Research, vol. 80, no. 10, pp. 1215 - 1221, 2010.

[15] N. Yang, D. Paire, F. Gao, A. Miraoui, and W. Liu, "Compensation of droop control using common load condition in DC microgrids to improve voltage regulation and load sharing," International Journal of Electrical Power \& Energy Systems, vol. 64, pp. 752 - 760, 2015.

[16] W. H. Kersting, Distribution system modeling and analysis. CRC press, 2012 